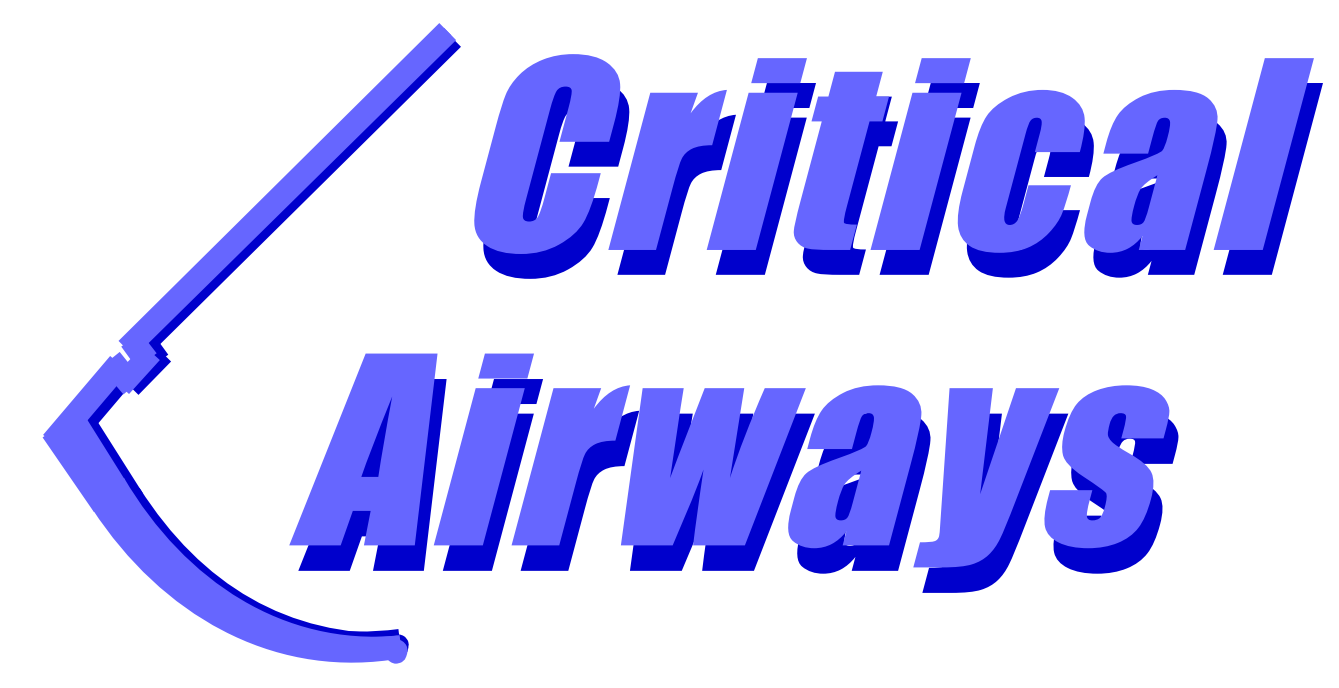

An educational initiative for professionals managing critically ill patients" airways

\author{
Aiyathurai S. ${ }^{1}$, Stephen L.2, Dasan J.1 \\ ${ }^{1}$ Kings College Hospital NHS Trust, Dept of Anaesthesiology, London, \\ United Kingdom, ${ }^{2}$ Kings College Hospital NHS Trust, Dept of Intensive \\ Care, London, United Kingdom
}

The "Critical Airways" training programme was developed at King's College Hospital (London) in response to perceived threats to patient safety. Airway procedures are traditionally viewed as the remit of anaesthetists, however the national audit carried out by Cook et al. indicated that adverse airway incidents also involved other healthcare professionals who usually receive much less training in airway management ${ }^{1}$. Non-anaesthetists were often in a position to prevent or mitigate the consequences of airway complications, and further training of all groups of professionals was recommended.

The airway management of critically ill patients is complicated by the unplanned or emergency nature of interventions, as well as the grossly abnormal physiology of critically ill patients. The main objective of the "Critical Airways" programme is to improve the practical airway skills of students, with an emphasis on improving safety in the event of airway emergencies. Although the training is aimed at non-anaesthetists, it does seek to reinforce the existing airway management skills of candidates so that they may employ basic techniques in clinical practice, whilst also recognising the limits of their expertise.

\section{Educational Interventions}

A one day training course was developed after conducting some preliminary training sessions. The morning session consisted of a number of tutorials on the following key topics:

- Complications of Airway Management Identification of the factors linked to airway complications in the national audit project ${ }^{1}$

- Non Technical Skills Identification of the cognitive, social and personal resource skills that complement technical skills, and contribute to safe task performance

- Risk Management Assessment of clinical risk in terms of severity of illness and airways risk

- Effects of Anaesthesia Consideration of the effects of critical illness on the response to anaesthesia

- Difficult Airways Recognition of the increased incidence of difficult airways in critical care; description of strategies for difficult ventilation and difficult intubation

During the tutorials, pre-recorded videos of clinical scenarios involving airway emergencies in simulated critically ill patients were viewed (refer to fig 1) The afternoon session consisted of five practical stations arranged in a circuit. Candidates were assigned to small groups with other candidates of a similar background. The stations were designed to address five main airway skill sets in Critical Care
- Basic Airway Management Identification of signs of airway obstruction; performance of basic airway manoeuvres

- Intubation Preparation for emergency intubation; assisting with simulated emergency intubation

- Difficult Airways Description of secondary intubation techniques; assisting with ventilation and maintenance of oxygenation

- Extubation Assessment for extubation; description of complications of extubation; assisting with simulated reintubation

- Tracheostomy Management ${ }^{2}$ Initial assessment of an unwell patient with a tracheostomy; identification of tracheostomy "red flags"

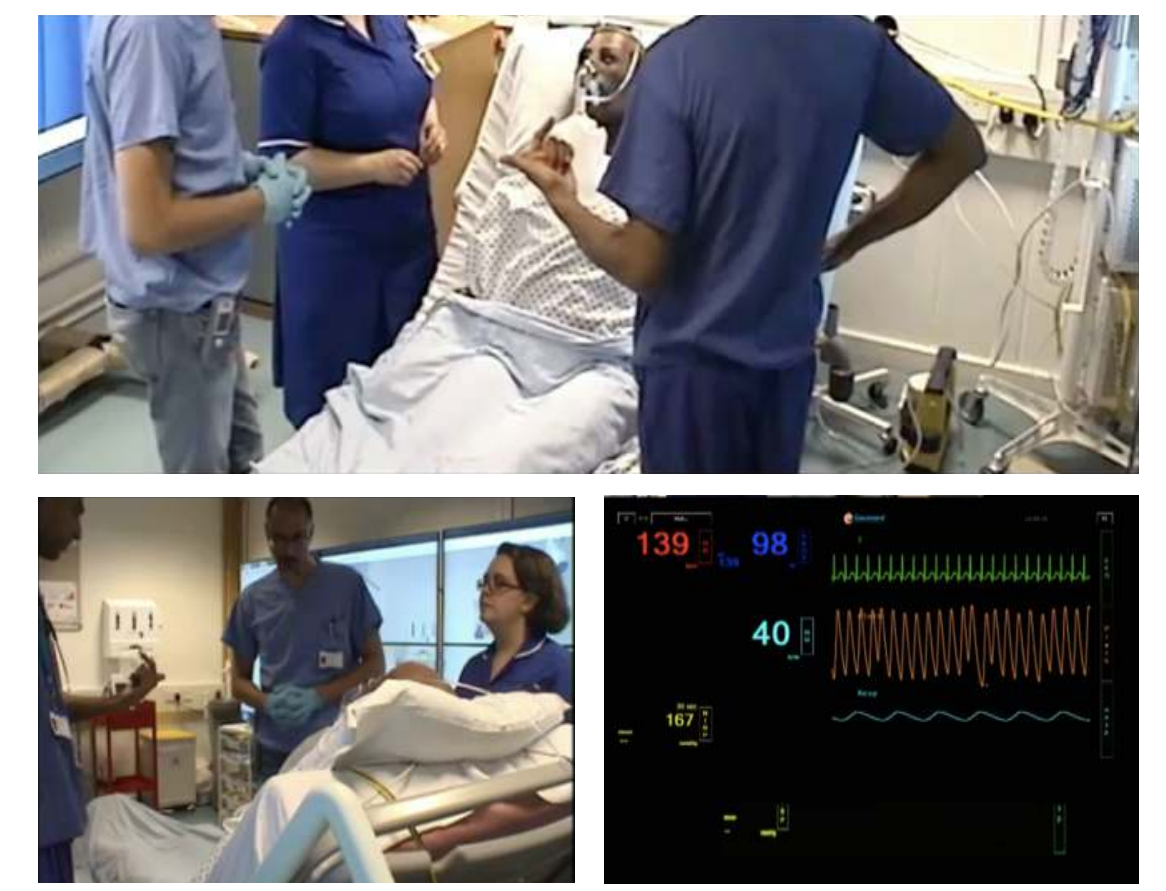

Fig 1: Images from video of a simulated airway emergency

\section{Evaluation}

Three days of training were conducted between May 2015 and March 2016. A total of 39 candidates completed the Critical Airways training course. The feedback from 38 candidates is detailed is summarised in the table below

Question

Mean Score (scale 1 - 9)

The objectives of the course were met

My knowledge of airway management has improved

My practical skills have improved

8.56

My management of airway problems is safer

8.44

8.10

I received helpful feedback

8.33

8.38

The trainers were supportive

8.92

I would recommend this training to my colleagues $\quad 8.77$

\section{Conclusions}

In terms of the educational impact of the course, the participants scored it most highly in respect of meeting the course objectives, supportiveness of the trainers and willingness to recommend the training to others. However the overall objective of improving patient safety is difficult to achieve through an educational course delivered on a single day. Although there may be shifts in attitude for individual participants after undergoing training, it should be recognised that patient safety is dependent on a range of broader institutional factors ${ }^{3}$. We suggest that when managing the most ill patients, even a single intervention to improve the skill set of healthcare practitioners may prove critical.

\section{References}

1. Cook, T.M. Woodall N. et al (2011) Major complications of airway management in the UK: results of the 4th National Audit Project of the Royal College of Anaesthetists and the Difficult Airway Society. British Journal of Anaesthesia 106 (5) 632-642

2. McGrath, B.A., Bates, L., et al (2012), Multidisciplinary guidelines for the management of tracheostomy and laryngectomy airway emergencies. Anaesthesia 67: 1025-1041

3. Vincent $C$ (2003) Understanding and responding to adverse events. New England Journal of Medicine 348 (11) 1051-6 